\title{
On the super-Rayleigh/subseismic elastodynamic indentation problem
}

\author{
H.G. GEORGIADIS ${ }^{1}$ \& J.R. BARBER ${ }^{2}$ \\ ${ }^{1}$ School of Technology, Box 422, The Aristotle University of Thessaloniki, 54006, Greece \\ ${ }^{2}$ Department of Mechanical Engineering and Applied Mechanics, University of Michigan, Ann \\ Arbor, MI 48109-2125, USA
}

Received 12 February 1991; accepted 10 June 1991

\begin{abstract}
The elastodynamic super-Rayleigh/subseismic indentation paradox is examined in this paper. Both the Craggs/Roberts steady-state problem and the Robinson/Thompson transient problem are reconsidered. Certain features of these solutions are discussed from a new point of view, by considering asymptotics at the end of the contact region, the influence of contact inequalities, energetics of the process and existence/uniqueness.
\end{abstract}

\section{Introduction}

A problem of considerable interest in elastodynamics concerns the steady motion of a frictionless rigid indenter at velocity $V$ across the surface of an elastic half-plane, as shown in Fig. 1. In the case where the indenter is a cylinder of radius $R$, this problem is the elastodynamic counterpart of the classical Hertzian contact problem, but the more general case of an arbitrary indenter presents no special additional problems.

Craggs and Roberts [1] obtained solutions for the sub-Rayleigh case $\left(V<c_{R}\right)$ and the superseismic case $\left(V>c_{1}\right)$, where $c_{1}=\sqrt{(\lambda+2 \mu) / \rho}, c_{2}=\sqrt{\mu / \rho}$ are the dilatational and shear wave speeds respectively, $\lambda, \mu$ are Lamé's constants, $\rho$ is the density, and $c_{R}$ is the Rayleigh wave speed defined by the equation $R\left(c_{R}\right)=0$, where [2]

$$
R(V) \equiv\left(2-M_{2}^{2}\right)^{2}-4 \sqrt{\left(1-M_{1}^{2}\right)\left(1-M_{2}^{2}\right)}
$$

and

$$
M_{1}=\frac{V}{c_{1}} ; \quad M_{2}=\frac{V}{c_{2}}
$$

However, they were unable to find physically acceptable solutions in the range $c_{R}<V<c_{1}$, and 26 years later there are still no satisfactory hypotheses as 


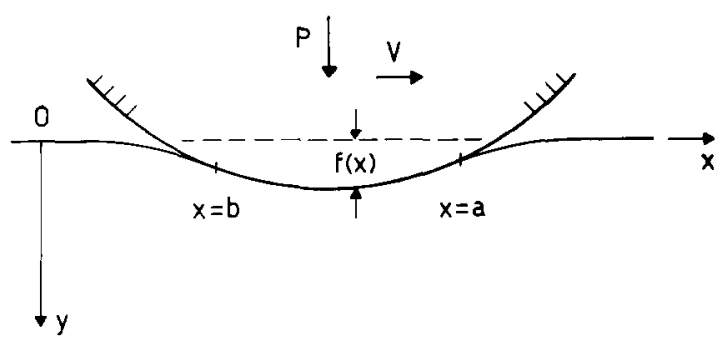

Fin. 1. The moving punch problem.

to what would happen to an indenter driven across a half-plane in this speed range. The present paper sets out to clarify certain features of the paradox and to demonstrate connections with other problems with similar features. We also hope it will lead to a reawakening of interest in this challenging problem, which constitutes a major gap in the completeness of classical linear elastodynamics.

The organization of the paper is as follows. Section 2 contains a discussion of the steady-state moving punch problem, using an integral equation formulation, with particular reference to the conditions at the end points and the contact inequalities. Section 3 presents energy and uniqueness considerations for the same problem. Section 4 is devoted to the transient Robinson/Thompson indentation problem and common features of all such problems and possible resolutions are discussed in Section 5.

\section{The moving punch problem}

If we assume (i) that the problem of Fig. 1 has a steady-state solution in which the stress fields are invariant in a frame of reference moving with the indenter and (ii) that the contact area is a single connected region defined by $b<x<a$ in this frame of reference, we can reduce the problem to a classical elastodynamic boundary-value problem. A complex variable formulation is given by Eringen and Suhubi $[2, \S 7.13]$.

If the boundaries of the contact region $a, b$ are assumed known, the resulting boundary-value problem is well-posed for all $V$ and has a unique solution. However, the physical problem demands that $a$ and $b$ be determined as part of the solution from the unilateral or Signorini contact conditions which state that (i) the contact traction $p(x)$ should be non-tensile throughout the contact region and (ii) the gap $g(x)$ between the indenter and the surface of the half-plane should be non-negative throughout the non-contact or separation region, i.e.

$$
\begin{aligned}
& p(x) \geqslant 0 ; b<x<a, \\
& g(x) \geqslant 0 ; x<b \text { and } x>a .
\end{aligned}
$$


In static contact problems, these inequalities are sufficient to define a unique contact area, the corresponding uniqueness and existence theorems being proved by Fichera [3]. However, this result does not carry over to the steadystate elastodynamic problem, though, as we shall see below, the evidence of particular solutions suggests that corresponding theorems might be provable for $V<c_{R}$.

The contact pressure distribution $p(x)$ in the sub-Rayleigh range has the same semi-elliptic form as in the static Hertzian problem, being given by [2]

$$
p(x)=\frac{2 P}{a} \sqrt{1-\frac{x^{2}}{a^{2}}} ; \quad-a<x<a,
$$

where $P$ is the total normal force applied to the indenter and $a$ is the semi-width of the contact area, given by

$$
a=M_{2} \sqrt{-\frac{2 P R \sqrt{1-M_{1}^{2}}}{\pi \mu R(V)}},
$$

where we note that $R(V)<0$ in the range $0<V<c_{R}$.

The expression $\mu R(V)$ decreases monotonically with $V$ showing that the halfplane responds to the moving indenter like a static half-plane with a reduced modulus. Thus, if we keep $P$ constant and increase $V$, the contact width will increase, becoming unbounded as $V \rightarrow c_{R}$.

\subsection{The Green's function}

This behavior can be predicted from the appropriate Green's function, which corresponds to the problem of a point normal load $P$, moving at speed $V$ across the surface of the half-plane (see Fig. 2). This problem was first considered by

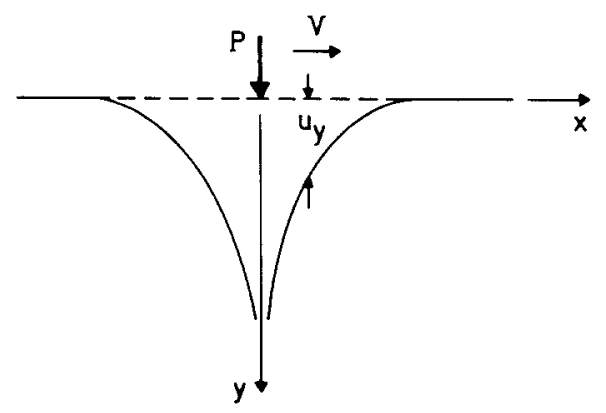

Fig. 2. Force moving over a half-plane. 
Cole and Huth [4] (see also $[2, \S 7.11]$ ). The normal surface displacement for this problem can be written in the generalized form ${ }^{1}$

$$
u_{y}(x, 0)=\frac{P}{\mu}\left[F_{1}(V) \log (r)+F_{2}(V) H(-x)\right]
$$

(omitting an arbitrary rigid body displacement), where $r=|x|$ is the distance from the moving load, $H$ is the Heaviside step function and

$$
\begin{aligned}
F_{1}(V) & =\frac{M_{2}^{2} \sqrt{1-M_{1}^{2}}}{\pi R(V)} ; \quad V<c_{2} \\
& =\frac{M_{2}^{2}\left(2-M_{2}^{2}\right)^{2} \sqrt{1-M_{1}^{2}}}{\pi\left[\left(2-M_{2}^{2}\right)^{4}+16\left(1-M_{1}^{2}\right)\left(M_{2}^{2}-1\right)\right]} ; c_{2}<V<c_{1} \\
& =0 ; V>c_{1}, \\
F_{2}(V) & =0 ; V<c_{2} \\
& =\frac{4 M_{2}^{2}\left(1-M_{1}^{2}\right) \sqrt{M_{2}^{2}-1}}{\left(2-M_{2}^{2}\right)^{4}+16\left(1-M_{1}^{2}\right)\left(M_{2}^{2}-1\right)} ; c_{2}<V<c_{1} \\
& =\frac{M_{2}^{2} \sqrt{M_{1}^{2}-1}}{\left(2-M_{2}^{2}\right)^{2}+4 \sqrt{\left(M_{1}^{2}-1\right)\left(M_{2}^{2}-1\right)}} ; V>c_{1} .
\end{aligned}
$$

In the range $V<c_{R}$, this expression is of the same form as the corresponding static Green's function ${ }^{2}$, differing only in the multiplying constant, which can be interpreted as a dynamic compliance or inverse modulus. It follows that any static indentation solution for the half-plane has a sub-Rayleigh elastodynamic counterpart of the same form, which can be recovered simply by replacing the static modulus by the equivalent dynamic modulus. In particular, we note that this equivalent modulus tends to zero at $V=c_{R}$, which can be seen as a resonance of the system.

The Green's function preserves the same form in the speed range $c_{R}<V<c_{2}$, but here we notice a surprising result - the Rayleigh polynomial $R(V)$ changes sign so that an inwardly directed moving load produces an outward displacement of the half-plane surface. This property is preserved in the transonic range $c_{2}<V<c_{1}$, but the Green's function then contains an additional step function

\footnotetext{
${ }^{1}$ Note that the expression for Cole and Huth's original expression for $F_{2}(V)$ in $c_{2}<V<c_{1}$ is in error by a factor of $M_{2}^{2} / 2$, this error being also present in the results given in [2]. The correct expression is given here and the full derivation is given in [5].
}

${ }^{2}$ Which of course can be recovered by allowing $V$ to tend to zero. 
term. The sign change in the Green's function at the Rayleigh wave speed might seem less surprising when considered in conjunction with the behaviour of a simple one degree of freedom dynamic system which of course vibrates $180^{\circ}$ out of phase with the force when excited above the resonant frequency.

It is interesting to note that the function $F_{1}(V)$ multiplying the singular (logarithmic) term in equation (2.5) is proportional to the instantaneous displacement at a distance $x$ from a normal impulse at $t=0$, where $x=V t$. This can be confirmed by comparing equation (2.6) with the corresponding results of $[2, \S 7.16]$. A curious feature of the function $F_{1}(V)$ is that it touches zero without changing sign at the particular speed $V=\sqrt{2} c_{2}$. The Green's function then has no singular term implying as we shall see below that the smooth punch problem has a very simple well-behaved solution for this speed, even though no such solution exists for infinitesimally higher or lower speeds.

\subsection{An integral equation formulation}

The Green's function of equation (2.5) permits a very efficient integral equation formulation of the problem. We can write down the normal surface displacement due to an arbitrary contact pressure, $p(x)$, in the form of a convolution integral, i.e.

$$
u_{y}(x)=\frac{1}{\mu} \int_{b}^{a}\left[F_{1}(V) \log |x-\xi|+F_{2}(V) H(\xi-x)\right] p(\xi) \mathrm{d} \xi .
$$

If $a, b$ are assumed given, the problem is now defined by the contact condition

$$
g^{\prime}(x) \equiv u_{y}^{\prime}(x)-f^{\prime}(x)=0 ; b<x<a,
$$

where $f(x)$ defines the shape of the punch and the condition is stated in differential form to avoid problems with logarithmically unbounded rigid body displacements.

Substituting (2.8) into (2.9), we obtain the classical Cauchy integral equation

$$
F_{1}(V) \int_{b}^{a} \frac{p(\xi) \mathrm{d} \xi}{(x-\xi)}-F_{2}(V) p(x)=\mu f^{\prime}(x) ; b<x<a
$$

for the unknown contact pressure distribution $p(x)$. In the special case where the indenter is a cylinder of radius $R$, the function $f^{\prime}(x)=-x / R$.

If neither of the functions $F_{1}(V), F_{2}(V)$ is zero, this is a singular integral equation of the second kind. However, if $V<c_{2}, F_{2}(V)$ is zero and the equation reduces to an integral equation of the first kind. Finally, if $V>c_{1}$ and also at the 
special value $V=\sqrt{2} c_{2}, F_{1}(V)=0$ and (2.10) reduces to an explicit algebraic equation for $p(x)$.

The solution of the integral equation (2.10) can be written as the sum of a particular integral and a homogeneous solution which contains an arbitrary constant. A convenient summary of the general solution is given by Johnson $[6, \$ 2.7]$. Both solutions are generally singular at both ends of the range, the strength of the singularity being described by the multiplier $(a-x)^{-1 / 2+\beta}(x-b)^{-1 / 2-\beta}$ where the real dimensionless parameter $\beta$ is defined by the equation

$$
\tan (\pi \beta)=\frac{F_{2}(V)}{\pi F_{1}(V)} ; 0<\beta<\frac{1}{2} .
$$

In particular, when the step function term $F_{2}(V)$ is zero, $\beta=0$ and we recover the classical square-root singularity.

The solution has to be chosen in such a way that (i) the inequalities $(2.1,2.2)$ are satisfied and (ii) the total load is a prescribed quantity, $P$, i.e.

$$
\int_{b}^{a} p(x) \mathrm{dx}=P
$$

In the static case, $V=0$, a consideration of the asymptotic fields near the end points [7] shows that the inequalities require that the contact pressure $p(x)$ be bounded at $a, b$ and hence that the multipliers on the two square-root singular terms be zero. These conditions and (2.12) then constitute three conditions for the arbitrary multiplier on the homogeneous solution of (2.10) and for the unknowns $a, b$. However, in view of what follows, we must emphasise that there is no physical objection to square-root singularities per se. The only reason for demanding boundedness in $p(x)$ is that otherwise one or other of the inequalities $(2.1,2.2)$ will be violated in the immediate vicinity of the end points.

\subsection{Conditions at the end points}

The asymptotic field near a discontinuity in boundary conditions is usually explored using the technique due to Williams [8], in which the fields are referred to a system of polar coordinates centered on the discontinuity and the two contacting bodies appear as semi-infinite wedges. This method has been extensively used by Comninou and Dundurs for thermoelastic contact problems $[9,10]$ and for problems involving Coulomb friction [11]. However, the corresponding results in the present problem can conveniently be obtained by performing a singular asymptotic analysis on the integral (2.8), making use of (2.11). 
Thus, in the range $0<V<c_{2}$, where $F_{2}(V)=0$, we find $\beta=0$ and hence the solution of equation (2.10) is generally square-root singular at both ends of the range. We can therefore define a function which is bounded at $x=a, b$ through the relation

$$
q(x)=p(x) \sqrt{(a-x)(x-b)}
$$

in which case the derivative of (2.8) can be written in the form

$$
u_{y}^{\prime}(x)=\frac{F_{1}(V)}{\mu} \int_{b}^{a} \frac{q(\xi) \mathrm{d} \xi}{(x-\xi) \sqrt{(a-\xi)(\xi-b)}}
$$

Since the singular behaviour of $p(x)$ has been described by the explicit multiplier $[(a-\xi)(\xi-b)]^{-1 / 2}$ in equation (2.14), the function $q(\xi)$ must be capable of a series expansion near $\xi=a$ in the form

$$
q(\xi)=\sum_{n=0}^{\infty} q_{n}(a-\xi)^{n}
$$

Substituting this expression into (2.14) and performing the integration, we obtain

$$
u_{y}^{\prime}(x)=\frac{\pi F_{1}(V)}{\mu} \sum_{n=0}^{\infty} q_{n}\left\{\sum_{i=1}^{n} \frac{(2 i-3) ! !}{(i-1) !}\left(\frac{a-b}{2}\right)^{i-1}(a-x)^{n-i}+(a-x)^{n} I_{0}\right\}
$$

where $(2 i-3) ! !=1.3 .5 \ldots(2 i-3)$ and

$$
\begin{aligned}
I_{0} & =-\frac{1}{\sqrt{(a-x)(b-x)}} ; x<b \\
& =0 ; b<x<a \\
& =\frac{1}{\sqrt{(x-a)(x-b)}} ; x>a
\end{aligned}
$$

\subsection{Consequences of the inequalities}

The discontinuity in (2.16) associated with the term involving $I_{0}$ implies a corresponding discontinuity in $g^{\prime}(x)$ and will lead to violation of the inequality (2.2) unless the first non-zero coefficient in (2.15) is of appropriate sign. The 
contact condition (2.9) requires $g^{\prime}(x)=0$ in $b<x<a$ and hence (2.2) requires that the discontinuity be such as to give $g^{\prime}\left(a^{+}\right)>0$.

If $q_{0} \neq 0$, this implies

$$
F_{1}(V), q_{0}>0
$$

but if $q_{0}=0$, the second order term in (2.16) is dominant, leading to the condition

$$
F_{1}(V), q_{1}<0 ; q_{0}=0
$$

However, the definition (2.13) implies that the first non-zero coefficient in (2.15) must be positive if the inequality (2.1) is to be satisfied, i.e.

$$
q_{0}>0 \text { or } q_{0}=0, q_{1}>0 .
$$

In the sub-Rayleigh range $0 \leqslant V<c_{R}, F_{1}(V)<0$ and hence (2.18-2.20) can only be satisfied by taking $q_{0}=0, q_{1}>0$. This leads to the familiar bounded square-root behaviour in the contact tractions and imposes uniqueness on the problem by providing a condition $\left(q_{0}=0\right)$ to determine the boundary of the contact area.

By contrast, in the range $c_{R}<V<c_{2}$, we find $F_{1}(V)>0$. It is then possible to satisfy $(2.18-2.20)$ by taking $q_{0}>0$, leading to a square-root singular traction distribution at $x=a$ and a discontinuous slope to the deformed surface. However, this deprives us of the condition for determining $a$ as well as implying the physically unlikely configuration of Fig. 3 .

It is tempting to demand boundedness in the contact tractions for its own sake (or for the sake of uniqueness), rather than as a consequence of the inequalities, but we then find that, if we set $q_{0}=0$, either sign for the next coefficient $q_{1}$ will lead to a violation of one of the two conditions $(2.19,2.20)$. In

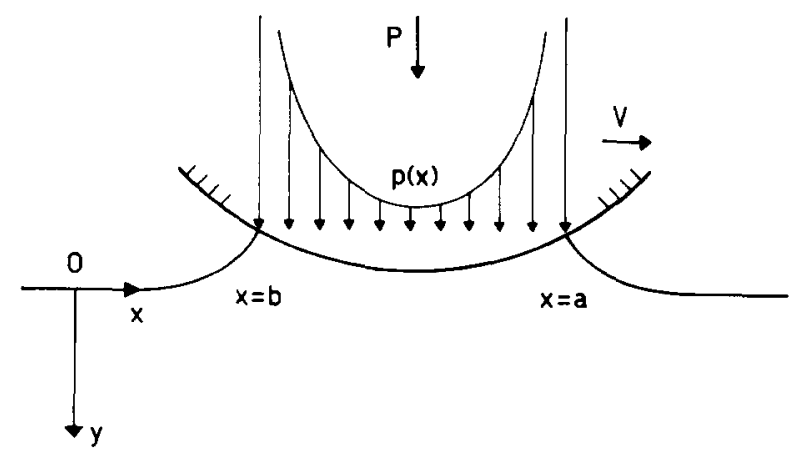

Fig. 3. Solution with singular tractions. 
other words, a smooth transition between contact and separation is impossible in the speed range $c_{R}<V<c_{2}$. Similar results can be established for the trailing edge of the contact region $x=b$.

The same argument can be extended to the speed range $c_{2}<V<c_{1}$, by replacing (2.13) by the more general form

$$
q(x)=p(x)(a-x)^{-1 / 2+\beta}(x-b)^{-1 / 2-\beta} .
$$

Once again, we find that, except at the special value $V=\sqrt{2} c_{2}$, a smooth transition from contact to separation violates the inequalities at either end of the contact region, whereas a singular transition satisfies them if the multiplier is of appropriate sign.

For completeness, we also consider the case where $V>c_{1}$ or $V=\sqrt{2} c_{2}$, for which equation (2.10) reduces to the explicit algebraic equation

$$
p(x)=-\frac{\mu f^{\prime}(x)}{F_{2}(V)} .
$$

The inequality (2.1) then requires $f^{\prime}(x) \leqslant 0$ in the contact area, since $F_{2}(V)>0$, whereas (2.2) demands that $g^{\prime}\left(a^{+}\right) \geqslant 0$ and $g^{\prime}\left(b^{-}\right) \leqslant 0$. These conditions will be satisfied near $x=a$, provided that $f^{\prime}(a) \leqslant 0$, but at $x=b$, they can only be simultaneously satisfied by choosing $b$ such that $f^{\prime}(b)=0$. Equation (2.12) then serves to determine the point $a$ and imposes uniqueness on the problem.

\section{Objections to the singular tractions}

The above discussion shows that the contact inequalities $(2.1,2.2)$ do not preclude singular contact tractions at the edges of the contact region when the speed is in the ranges $c_{R}<V<\sqrt{2} c_{2}$ and $\sqrt{2} c_{2}<V<c_{1}$-indeed, they preclude bounded tractions in this range. Should we therefore conclude that the resulting singular solutions describe the behaviour of the physical system?

There are two objections to this conclusion, one concerning the energy flux at the singular point and the other uniqueness.

\section{I. Energy considerations}

When a crack in a homogeneous material propagates at a speed $V<c_{R}$, energy is absorbed at the crack tip at a rate dependent on $V$. This can be demonstrated by calculating the energy flux across a contour enclosing the crack tip and then letting the dimensions of the enclosed region tend regularly to zero [12]. In the 
limit as $V \rightarrow 0$, this result reduces to the familiar energy release rate calculation used in quasi-static fracture mechanics. It is reasonable to assume that the energy in question supplies the surface energy of the newly generated crack faces.

A similar argument shows that energy will be emitted at the singular point when a square-root singular distribution of normal tractions such as that at $x=a$ in Fig. 3 moves to the right over the surface of the half-plane - in fact, the stress fields in the two cases are identical except for a sign change, since symmetry in the fracture problem ensures that the unfractured region of the crack plane remains plane. However, it is now less clear what physical mechanism is responsible for this energy production.

Freund [13] uses this argument to cast doubt on the physical occurrence of a class of interface waves involving moving separation regions analyzed by Comninou and Dundurs [14]. Interface waves at unilateral interfaces are known to be possible for certain material combinations [15] and if the amplitude of such a wave exceeds the mean interface pressure, it seems reasonable to anticipate travelling regions of separation and contact. However, Comninou and Dundurs showed that steady-state solutions of this form are only possible if square-root singular tractions are admitted. In a later paper [16], they also showed that a disturbance of a more general form could propagate at a frictional interface without slip, under the same assumptions.

Fracture and wave propagation problems exhibit considerable mathematical similarities with dynamic contact problems, but there is one very important physical difference - the velocity in a contact problem is an independent variable, whereas in fracture or wave propagation it is a dependent variable. If we do a fracture experiment, we have to accept the crack propagation rate corresponding to the applied stress or displacement conditions, but, in principle at least, there is nothing to stop us from conducting a dynamic contact problem in which the indenter has any desired speed $V$. This is why the non-existence of acceptable solutions to such problems is such a pressing question. What would happen if we tried to drive a massive rigid vehicle over a large body above the Rayleigh wave speed?

Any argument about the strength of acceptable singularities in contact problems must accommodate the fact that the contact tractions at the edge of a rigid punch with a sharp corner are square-root singular. In their response to Freund's objections, Comninou and Dundurs [17] suggest that we might regard the sharp punch as the limiting case of a slightly rounded punch as the radius at the corner tends to zero. For a finite corner radius, the contact tractions would then be everywhere bounded, but (i) they would have a maximum near the edge of the contact region and (ii) contact would extend slightly around the corner, so that the tractions in this region would do work on the half-plane as the punch moved. It is therefore plausible that the total work done by the tractions in this small region would tend to a constant as the corner radius tended to zero. 
Notice also that work would be done on the punch by the tractions in the corresponding region at the trailing edge, which is consistent with the fact that the corresponding singularity appears as an energy sink.

Unfortunately, this argument fails when $c_{R}<V<c_{2}$, since, as we have seen in Section 2 above, making the punch rounded does not then enable us to dispose of the singular tractions. The problem is even more severe in the range $c_{2}<V<c_{1}$, since the singularity at the trailing edge $x=b$ is then stronger than square-root and the corresponding energy flux across a contour surrounding the singular point increases without limit as the contour is shrunk to zero. This seems to imply that the singularity constitutes an infinite energy sink, which is surely physically unacceptable. However, we should remark that this supposedly infinite supply of energy does not come from the half-plane, since the flux across all contours of finite length remains bounded at all times, whether or not the contour includes the singular point. Another curious result is that we obtain an infinite energy flux in the limit, even where the contour does not include the singular point, provided that we shrink the contour to zero in a self-similar way (e.g. the semi-circle of radius $c$ centered on the point on the surface a distance $A c$ ahead of the singularity, where $A$ is an arbitrary constant). Of course, all such points coincide with the singular point in the limit $c \rightarrow 0$, but they strictly never contain the supposed energy sink and hence the energy flux must be associated with a corresponding infinite rate of change of the total strain and kinetic energy of the material within the contour.

\subsection{Non-uniqueness}

The second criticism of singular traction distributions in smooth contact problems centers around the fact that when they are admitted, the solution exhibits non-uniqueness. In the problem of Fig. 1, the admission of singular tractions at the end of the contact region eliminates the condition required to determine the location of these points and infinitely many solutions can be obtained with different extents for the contact region.

Also, in the interface wave problem $[14,16]$, non-uniqueness is suggested by the fact that waves can be found which propagate at any speed within a given range, whereas in the absence of separation, there is a unique propagation speed. Non-uniqueness of solution is acceptable if it is associated with historydependence of solution and, in this context, Comninou and Dundurs [17] suggest that the wave propagation speed depends on conditions at the initiation of the disturbance. A similar dependence on initial conditions might be suggested for the moving punch problem, but questions of stability must inevitably be raised, particularly in view of the fact that the range of multiple solutions form a continuous sequence, so that only an infinitesimal disturbance would be needed to change the contact region from its initial value. 


\section{The Robinson and Thompson problem}

The difficulties outlined in the previous sections are not restricted to steady-state moving contact problems, but seem to arise in any elastodynamic problem in which a boundary between contact and separation is forced to move at a speed in the super-Rayleigh/subseismic range $c_{R}<V<c_{1}$. Thus, it is encountered in problems where a crack is forced to propagate at a given speed by a wedge $[18,19]$ and in the self-similar dynamic indentation of a half-space by a wedgeshaped indenter $[20,21]$.

Robinson and Thompson's solution [21] of the latter problem is of particular interest, since they accept the singularities at the edge of the contact region in the offending speed range, but succeed in obtaining a unique solution by demanding boundedness in the stress and displacement components in other regions of the half-plane. Their work is widely considered as classical, being cited in the standard elastodynamics texts $[2, \S 7.18]$ as well as in numerous papers on elastodynamic contact. It is therefore the more surprising that their resolution of the uniqueness question has received comparatively little critical attention, despite the fact that it is the only such resolution in the multitude of special problems exhibiting this peculiar difficulty and might therefore seem to hold out hope of a more general resolution. However, we shall argue in the present section that their procedure for obtaining uniqueness is erroneous.

\subsection{Statement of the problem}

The elastodynamic indentation problem is depicted in Fig. 4. A rigid frictionless wedge-shaped indenter of angle $\gamma$ is driven into an elastic half-plane at constant speed $V$, first contact occuring at the apex at time $t=0$. Dimensional considerations lead us to anticipate a self-similar solution in which the stress and displacement fields have a spatially invariant form in a dimensionless coordinate



Fig. 4. The Robinson/Thompson problem. 
system $x / V t, y / V t$ and the contact region expands at uniform velocity $a$. Many authors have investigated self-similar problems in elastodynamics, general treatments including extensive additional references being given by Eringen and Suhubi [2, §§7.14-7.19], Willis [22] and Georgiadis [23].

The boundary conditions for all $t>0$ can then be stated in the form

$$
\begin{aligned}
& u_{y}(x, 0, t)=V t-|x| \tan \left(\frac{\pi-\gamma}{2}\right) ;|x|<a t, \\
& \sigma_{y y}(x, 0, t)=0 ;|x|>a t, \\
& \sigma_{y x}(x, 0, t)=0 ; \text { all } x, \\
& u_{y}(x, 0, t)>V t-|x| \tan \left(\frac{\pi-\gamma}{2}\right) ;|x|>a t, \\
& \sigma_{y y}(x, 0, t)<0 ;|x|<a t,
\end{aligned}
$$

where $a$ is a constant to be determined. The half-plane is assumed to be at rest and stress-free at time $t=0$.

Many authors [20,24-29] have considered this problem in the sub-Rayleigh regime, $a<c_{R}$, for which the inequalities demand that the traction be bounded at $|x|=a t$ and lead to a unique solution. However, as in Section 2.4, it is found that the bounded solution violates the inequalities if $c_{R}<a<c_{1}$, but that infinitely many singular solutions can be found which satisfy them. Notice incidentally that it is the velocity of the contact boundary $a$ which determines the character of the problem, not the punch velocity $V$, which will generally be much lower than $a$, since small strain considerations demand that the inclination of the wedge face $(\pi-\gamma) / 2 \ll 1$.

For a full description of Robinson and Thompson's solution method, the reader is referred to $[21]$ or $[2, \$ 7.18]$. The solution is developed in terms of two complex potentials $\theta_{1}, \theta_{2}$ which on the surface plane $y=0$ reduce to the common real form $\theta=\theta_{1}=\theta_{2}=t / x$. We shall only require the expressions for the stress and velocity components at this surface, which are written in the form

$$
\begin{aligned}
\sigma_{y y} & =\mathscr{R}\left[\sigma_{y y}^{*}(\theta)\right], \sigma_{x y}=\mathscr{R}\left[\sigma_{x y}^{*}(\theta)\right], \\
v_{y} & =\mathscr{R}\left[v_{y}^{*}(\theta)\right], v_{x}=\mathscr{R}\left[v_{x}^{*}(\theta)\right],
\end{aligned}
$$

where $\sigma_{y y}^{*}, \sigma_{x y}^{*}, v_{x}^{*}, v_{y}^{*}$ are complex functions of $\theta$ (see $[2, \S \S 7.14-7.19]$ ).

Further, the shear-traction-free condition (4.3) can be shown to require

$$
v_{y}^{* \prime}(\theta)=-\frac{\left(c_{1}^{-2}-\theta^{2}\right)^{1 / 2}}{\mu c_{2}^{2} R\left(\theta^{2}\right)} \sigma_{y y}^{* \prime}(\theta),
$$




$$
v_{x}^{* \prime}(\theta)=\frac{\theta}{\mu c_{2}^{2} R\left(\theta^{2}\right)} \sigma_{y y}^{* \prime}(\theta)
$$

where the transient Rayleigh function $R\left(\theta^{2}\right)$ is defined by

$$
R\left(\theta^{2}\right)=\left(c_{2}^{-2}-2 \theta^{2}\right)^{2}+4 \theta^{2}\left(c_{1}^{-2}-\theta^{2}\right)^{1 / 2}\left(c_{2}^{-2}-\theta^{2}\right)^{1 / 2} .
$$

By using these results, the equality boundary conditions $(4.1,4.2)$ can be written as

$$
\begin{aligned}
& \sigma_{y y}(x, 0, t)=\mathscr{R}\left(\int_{0}^{\theta} \sigma_{y y}^{* \prime} d \theta\right)=0 ;|\theta|<a^{-1}, \\
& v_{y}(x, 0, t)=\mathscr{R}\left(\int_{0}^{\theta} v_{y}^{* \prime} d \theta\right)=V ;|\theta|>a^{-1} .
\end{aligned}
$$

Robinson and Thompson [21] (see also [2, \$7.18]) established that the solution in the subsonic regime $a<c_{R}$ should have the general form

$$
\sigma_{y y}^{* \prime}(\theta)=i A_{1}\left(a^{-2}-\theta^{2}\right)^{-1 / 2}+i A_{2}\left(a^{-2}-\theta^{2}\right)^{-3 / 2},
$$

which emerges from the respective mixed boundary value problem of KeldyshSedov type. In this equation, $A_{1}, A_{2}$ are real constants and to satisfy (4.1), we must take

$$
A_{1}=\frac{4 \mu}{\pi}\left(1-\frac{c_{2}^{2}}{c_{1}^{2}}\right) \tan \frac{\pi-\gamma}{2}
$$

For $a<c_{R}$, it is found that the inequalities $(4.4,4.5)$ can only be satisfied by taking $A_{2}=0$, leading to the contact traction distribution

$$
\sigma_{y y}(x, 0, t)=-\frac{4 \mu}{\pi}\left(1-\frac{c_{2}^{2}}{c_{1}^{2}}\right) \tan \left(\frac{\pi-\gamma}{2}\right) \cosh ^{-1}\left(\frac{a t}{|x|}\right) ;|x|<a t,
$$

a result confirmed by other researchers [25-27].

\subsection{The range $c_{R}<a<c_{2}$}

We now turn our attention to indentation speeds leading to a contact boundary which moves at a speed in the range between $c_{R}$ and $c_{2}$. In this range, $A_{1}$ is still given by (4.13), but the inequality conditions no longer define a unique value for $A_{2}$. 
However, the Rayleigh function $R\left(\theta^{2}\right)$ defined by (4.9) has a zero at $\theta=c_{R}^{-1}$ and hence the complex functions $v_{y}^{* \prime}(\theta), v_{x}^{* \prime}(\theta)$ of $(4.7,4.8)$ will exhibit a pole at this value, unless the function $\sigma_{y y}^{* \prime}(\theta)$ has a cancelling zero. Robinson and Thompson [21] argued that such a pole was unacceptable and therefore explicitly introduced the factor $\left(c_{R}^{-2}-\theta^{2}\right)$ in their definition of $\sigma_{y y}^{* \prime}(\theta)$. Equation (4.12) remains valid for $c_{R}<a<c_{2}$, so their procedure is equivalent to choosing

$$
A_{2}=A_{1}\left(c_{R}^{-2}-a^{-2}\right),
$$

which ensures that $\sigma_{y y}^{* \prime}\left(c_{R}^{-1}\right)=0$.

The important point to notice here is that Robinson and Thompson impose uniqueness on the solution by demanding that the functions $v_{y}^{* \prime}(\theta), v_{x}^{*^{\prime}}(\theta)$ be analytic in $|\theta|>a^{-1}$ and in particular at the Rayleigh points $x= \pm c_{R} t$, which are the points reached by a disturbance travelling at $c_{R}$ and originating at the point of initial contact at $t=0$.

However, we shall prove in the next section that this procedure is incorrect, since even if the offending pole remains in the complex expressions for $v_{y}^{* \prime}(\theta), v_{x}^{*^{\prime}}(\theta)$, no unacceptable singularities appear in the corresponding real expressions for the stress and velocity components.

\subsection{Analysis}

The Robinson/Thompson procedure would be justifiable if the singularity in the functions $v_{y}^{* \prime}(\theta), v_{x}^{* \prime}(\theta)$ at the Rayleigh points led to (a) violation of the boundary conditions and/or (b) singularities in the physical quantities which are unacceptable within the context of linear elasticity. In the present section, we shall demonstrate that for the regime $c_{R}<a<c_{2}$, the general solution (4.12) is satisfactory according to these criteria for all values of the constant $A_{2}$.

\subsubsection{The boundary conditions}

We examine the possible violation of the boundary conditions by evaluating the normal surface velocity

$$
v_{y}(x, 0, t)=-\frac{1}{\mu c_{2}^{2}} \mathscr{R}\left[\int_{0}^{\theta} \frac{\left(c_{1}^{-2}-\theta^{2}\right)^{1 / 2} \sigma_{y y}^{* \prime}(\theta) d \theta}{R\left(\theta^{2}\right)}\right] \equiv-\frac{1}{\mu c_{2}^{2}} \mathscr{R}(I)
$$

from equations $(4.6,4.7)$.

This velocity must be non-singular at every point beneath the punch, since it is in fact equal to the indentation velocity $V$. We can easily show that $v_{y}(x, 0, t)$ is non-singular despite the fact that $v_{y}^{* \prime}(\theta)$ is singular at the Rayleigh points $\theta= \pm c_{R}^{-1}$.

Figure 5 shows the half-plane surface in the inverse velocity domain. When 


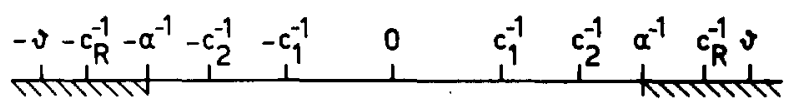

Fig. 5. The domain of integration for $\theta$ (the inverse velocity) in equation (4.16).

$|\theta|>a^{-1}$, the reference position is within the contact length and the integral $I$ in (4.16) can be decomposed into

$$
\begin{aligned}
I \equiv & I_{1}+I_{2}+I_{3}+I_{4} \equiv \int_{0}^{c_{1}^{-1}} F(\theta) d \theta+\int_{c_{1}^{-1}}^{c_{2}^{-1}} F(\theta) d \theta \\
& +\int_{c_{2}^{-1}}^{a^{-1}} F(0) d \theta+\int_{a^{-1}}^{\theta} F(\theta) d \theta
\end{aligned}
$$

where

$$
F(\theta)=\frac{\left(c_{1}^{-2}-\theta^{2}\right)^{1 / 2}\left[i A_{1}\left(a^{-2}-\theta^{2}\right)^{-1 / 2}+i A_{2}\left(a^{-2}-0^{2}\right)^{-3 / 2}\right]}{\left(c_{2}^{-2}-2 \theta^{2}\right)^{2}+4 \theta^{2}\left(c_{1}^{-2}-\theta^{2}\right)^{1 / 2}\left(c_{2}^{-2}-\theta^{2}\right)^{1 / 2}} .
$$

The singular point of $F(\theta)$ occurs at $\theta=c_{R}^{-1}$, which falls in the range of the integral $I_{4}$. However, it is clear from $(4.17,4.18)$ that $I_{4}$ is an imaginary quantity and hence it makes no contribution to the expression (4.16) for $v_{y}(x, 0, t)$. It follows that $v_{y}$ is not singular at the Rayleigh points and hence that the boundary conditions are not violated.

\subsubsection{Inadmissible singularities}

We next examine the other velocity component $v_{x}(x, 0, t)$ to see whether the general solution possesses any unacceptable singularities which do not affect the satisfaction of the boundary conditions. From $(4.6,4.8)$, we have

$$
v_{x}(x, 0, t)=\frac{1}{\mu c_{2}^{2}} \mathscr{R}\left[\int_{0}^{\theta} \frac{\theta \sigma_{y y}^{* \prime}(\theta) d \theta}{R\left(\theta^{2}\right)}\right] \equiv \frac{1}{\mu c_{2}^{2}} \mathscr{R}(J)
$$

For $c_{R}<a<c_{2}$, the domain of integration is again given by Fig. 5 and we can decompose $J$ into

$$
\begin{aligned}
J \equiv & J_{1}+J_{2}+J_{3}+J_{4} \equiv \int_{0}^{c_{1}^{-1}} H(\theta) d \theta+\int_{c_{1}^{-1}}^{c_{2}^{-1}} H(\theta) d \theta \\
& +\int_{c_{2}^{-1}}^{a^{-1}} H(\theta) d \theta+\int_{a^{-1}}^{\theta} H(\theta) d \theta,
\end{aligned}
$$


where

$$
H(\theta)=\frac{\theta\left[i A_{1}\left(a^{-2}-\theta^{2}\right)^{-1 / 2}+i A_{2}\left(a^{-2}-\theta^{2}\right)^{-3 / 2}\right]}{\left(c_{2}^{-2}-2 \theta^{2}\right)^{2}+4 \theta^{2}\left(c_{1}^{-2}-\theta^{2}\right)^{1 / 2}\left(c_{2}^{-2}-0^{2}\right)^{1 / 2}}
$$

The singular point $\theta= \pm c_{R}^{-1}$ is contained in the range of $J_{4}$, which now is a real quantity and which therefore does contribute to the function $v_{x}(x, 0, t)$ (see equations $(4.20,4.21)$ ). However, the singularity in the tangential velocity (and hence also in the stress component $\left.\sigma_{x x}(x, 0, t)\right)$ is logarithmic and is therefore acceptable within the context of linear elasticity.

It might be argued that, even though logarithmic singularities are admissible, the occurrence of such a singularity has no obvious physical cause and hence that Robinson and Thompson's solution which eliminates it is more physically plausible. In this context we note that a disturbance at the Rayleigh points can be interpreted as a disturbance originating at the first point of impact $(x=0$, $y=0, t=0$ ) and propagating with unchanged form at the Rayleigh wave velocity in both directions. Since $t=0$ is a point of discontinuity in the history of the process, we have no reason to preclude the occurrence of such a disturbance.

Perhaps a more cogent argument is that the sub-Rayleigh solution, which has been independently obtained and discussed by numerous authors [20, 24-29], also contains a disturbance of the same form in the vertical velocity $v_{y}(x, 0, t)$ at the Rayleigh points, which of course are now outside the contact area. However, as we have shown in Section 4.3.1, the disturbance does not affect the satisfaction of the contact boundary conditions and hence if it is deemed to be unacceptable, it must be equally unacceptable when it occurs outside the contact region. Furthermore, we cannot eliminate the disturbance in the subsonic case whilst still satisfying the inequality conditions-notably at the transition from contact to separation.

\subsection{The transonic regime}

An essentially similar argument may be developed for the transonic regime $c_{2}<a<c_{1}$. The general solution of the boundary value problem is now more complex, but it shares with equation (4.13) and with the solution of the Craggs/Roberts problem the characteristic of containing a term related to the punch profile which leads to a traction distribution bounded at the ends and a second term leading to singular tractions which contains an arbitrary multiplier. As before, Robinson and Thompson's procedure leads them to choose this second term so as to cancel the pole at the Rayleigh points in $v_{y}^{* \prime}(\theta)$ and hence impose uniqueness on the solution.

We also note that the strength of the singularity in the contact tractions is less 
than square root, being of the form $\left(a^{2}-x^{2}\right)^{-1 / 2+\beta}$ where $\beta$ is given by equation (2.11) with $V$ replaced by the velocity of the contact boundary $a$.

\section{Discussion}

We must conclude from the preceding arguments that the Robinson/Thompson procedure of enforcing uniqueness by requiring that the complex function $v_{y}^{* \prime}(\theta)$ be analytic at the Rayleigh points is unjustified. This conclusion imposes some consistency on the existing body of analytical solutions to elastodynamic contact problems, all of which share the following features:

- If the velocity $V$ of the transition point from contact to separation lies in the range $V<c_{R}, V>c_{1}$ or $V=\sqrt{2} c_{2}$, the Signorini inequalities are necessary and sufficient to determine a unique solution to the problem, including the determination of the extent of the contact region. The elastodynamic problem is then well posed.

- If singular contact tractions at the edge of the contact region are ruled out $a$ priori, no solutions exist in which this edge moves at a speed $V$ in the range $c_{R}<V<\sqrt{2} c_{2}$ or $\sqrt{2} c_{2}<V<c_{1}$.

- If, on the other hand, such singular tractions are admitted, the corresponding contact problem will have multiple solutions satisfying the inequalities for $c_{R}<V<\sqrt{2} c_{2}$ or $\sqrt{2} c_{2}<V<c_{1}$.

- If the edge of the contact region is advancing at speed $V$ into a region which was previously separated, the strength of the singularity will be equal to or weaker than square-root, being described by the multiplier $(a-x)^{-1 / 2+\beta}$, where $\beta$ is given by (2.11). However, if the edge is receding at speed $V-$ i.e. if contact is giving way to separation - the strength of the singularity will be equal to or stronger than square-root, being described by the multiplier $(x-b)^{-1 / 2-\beta}$. We note that an expanding crack $[30,31]$ is equivalent to a receding contact region and therefore has the same asymptotic behaviour ${ }^{3}$.

In the offending speed range, both alternatives seem equally unacceptable.

\subsection{Some speculations}

As we remarked earlier, indentation problems present the paradox more starkly than applications involving fracture or interface waves, since the punch velocity is an independent variable. However, we should remark that we cannot control

\footnotetext{
${ }^{3}$ It is worth noting that the error in Cole and Huth's results demonstrated in [5] was discovered as a result of an apparent lack of consistency between the predictions of equation (2.11) and the asymptotic behaviour observable in other elastodynamic contact and fracture problems.
} 
the velocity of the transition from contact to separation directly. We only appear to control it with the velocity of the punch when we make the assumption that the Craggs/Roberts problem has a solution which is invariant in a moving frame of reference or that the Robinson/Thompson problem has a self-similar solution.

What are the alternatives to these assumptions? There are precedents in other branches of mechanics for nominally steady systems to have unsteady solutions-for example, the frictional vibrations which can arise when the friction coefficient between two sliding bodies is a decreasing function of sliding velocity.

A conceivable unsteady scenario for the Craggs/Roberts problem would be for the contact area to move more slowly than the punch, so that it would tend towards the trailing edge. If the applied force was constant, this would permit the punch to move towards the half-plane until a new, disconnected contact area formed near the center of the punch. In effect the punch might appear to ride on a series of corrugations on the half-plane. In the same way, a non-self-similar solution to the Robinson/Thompson problem might be envisaged involving several disconnected contact areas. Such unsteady solutions would permit the systems to evolve, without requiring a transition from contact to separation ever to move at a velocity greater than $c_{R}$. The resulting behaviour may be quasiperiodic or chaotic in nature. It is perhaps not irrelevant to note that chaotic behaviour can occur in extremely simple one-dimensional systems when dynamic systems involve unilateral interfaces [32].

These arguments suggest that the paradox might be resolved by solving an appropriate transient problem - for example, an initially static Hertzian contact between a rigid cylinder and a plane, which starts to move at time $t=0$. Unfortunately, such problems are generally not tractable by analytical methods unless some fairly simple assumption can be made about the evolution of the contact area, which would destroy the purpose of the solution in the present instance. Numerical solutions are also very challenging in such problems, since waves will be generated by the discontinuities due to the discretization.

Aboudi [33] gives a finite difference formulation appropriate for problems of this type and gives some results for the wedge indentation problem, including one case in the transonic regime. His results for the extent of the contact area are fairly close to those of Robinson and Thompson and therefore cast some doubt on the arguments of Section 4 above. However, the results cannot be regarded as conclusive, since the true solution may involve features on a scale smaller than the discretization used. In particular, we note that violations of the inequalities associated with the asymptotic fields at the edge of the contact region (see Section 2.4) might occur in a very small region and hence require a very fine mesh for their resolution. It is also worth noting that the variational principle used in most numerical methods will tend to favour solutions without singularities when extra degrees of freedom are present. 


\section{Conclusions}

The present paper clearly raises many more questions than it solves, but these questions are very pressing and are apparently being neglected by the elastodynamic community. We shall have achieved our objective if we succeed in generating sufficient interest in this challenging problem to ensure that some progress will be made in the next 26 years.

\section{Acknowledgements}

J.R. Barber is pleased to acknowledge support from the National Science Foundation under Contract number MSM-8820623.

\section{References}

1. J.W. Craggs and A.M. Roberts, On the motion of a heavy cylinder over the surface of an elastic half-space. ASME J. Appl. Mech. 34 (1967) 207-209.

2. A.C. Eringen and E.S. Suhubi, Elastodynamics, Vol. Il. New York: Academic Press (1975).

3. G. Fichera, Problemi Elastostatici con Vincoli Unilaterali: il Problema di Signorini con Ambingue Condizioni al Contorno. Mem. Accad. Nax. Lincei, Ser. 8, 7 (1964 91-140.

4. J.D. Cole and J.H. Huth, Stresses produced in a half-plane by moving loads. ASME J. Appl. Mech. 25 (1958) 433-436.

5. H.G. Georgiadis and J.R. Barber, Steady-state transonic motion of a line load over an elastic half-space (The corrected Cole/Huth solution) ASME J. Appl. Mech. to appear (1993).

6. K.L. Johnson, Contact Mechanics, Cambridge University Press, Cambridge, (1985).

7. J. Dundurs and M. Comninou, Some consequences of the inequality conditions in contact and crack problems. J. Elasticity 9 (1979) 71-82.

8. M.L. Williams, Stress singularities from various boundary conditions in angular corners of plates in extension. ASME J. Appl. Mech. 19 (1952) 526-528.

9. M. Comninou and J. Dundurs, On the Barber boundary conditions for thermoelastic contact. ASME J. Appl. Mech. 46 (1979) 849-853.

10. M. Comninou and J. Dundurs, Thermoelastic contact involving a sharp corner. Wear 59 (1980) $53-60$.

11. M. Comninou, Stress singularity at a sharp edge in contact problems with friction. $Z$. Angew. Math Phys. 27 (1976) 493-499.

12. L.B. Freund, Dynamic crack propagation. In: F. Erdogan (ed.), The Mechanics of Fracture, New York: ASME (1976) pp. 105-134.

13. L.B. Freund, Discussion on reference [14]. ASME J. Appl. Mech. 45 (1978) 226-227.

14. M. Comninou and J. Dundurs, Elastic interface waves involving separation. ASME J. Appl. Mech. 44 (1977) 222-226.

15. J.D. Achenbach and H.I. Epstein, Dynamic interaction of a layer and a half-space. ASCE J. Eng. Mech. 93 (1967) 27-42.

16. M. Comninou and J. Dundurs, Can two solids slide without slipping? Int. J. Solids Structures 14 (1978) 251-260.

17. M. Comninou and J. Dundurs, Authors closure to [13]. ASME J. Appl. Mech. 45 (1978) 227 228. 
18. G.I. Barenblatt and R.V. Goldstein, Wedging of an elastic body by a slender wedge moving with a constant super-Rayleigh subsonic velocity. Int. J. Fracture 8 (1972) 427-434.

19. L.M. Brock, The non-uniform motion of a thin smooth rigid inclusion through an elastic solid. Q. Appl. Math. 36 (1978) 269-277.

20. R.J. Bedding and J.R. Willis, The dynamic indentation of an elastic half-space. J. Elasticity 3 (1973) 289-309.

21. A.R. Robinson and J.C. Thompson, Transient stresses in an elastic half-space resulting from the frictionless indentation of a rigid wedge-shaped die. ZAMM 54 (1974) 139-144.

22. J.R. Willis, Self-similar problems in elastodynamics. Phil. Trans. Roy. Soc. (London), 274 (1973) $435-471$.

23. H.G. Georgiadis, An integral equation approach to self-similar plane elastodynamic crack problems. J. Elasticity 25 (1991) 17-30.

24. B.V. Kostrov, Self-similar dynamic problems of pressing of a rigid die into an elastic half-space. Mekhanika i Mashinostroenie 4 (1964) 54-61.

25. E.F. Afanas'ev and G.P. Cherepanov, Some dynamic problems of the theory of elasticity. $J$. Appl. Math. Mech. (PMM), 37 (1973) 584-606.

26. G.P. Cherepanov, Mechanics of Brittle Fracture. McGraw-Hill, New York (1979).

27. L.M. Brock, Symmetrical indentation over a uniformly expanding contact region-I Basic analysis. Int. J. Engng. Sci. 14 (1976) 191-199.

28. L.M. Brock, Dynamic rigid indentation induced by sliding frictionless contact. Int. J. Engng. Sci. 16 (1978) 545-550.

29. L.M. Brock, Symmetrical indentation over a uniformly expanding contact region-II Perfect adhesion. Int. J. Engng. Sci. 15 (1977) 147-155.

30. H.G. Georgiadis, On the stress singularity in steady-state transonic shear crack propagation. Int. J. Fracture 30 (1986) 175-180.

31. L.B. Freund and R.J. Clifton, On the uniqueness of plane elastodynamic solutions for running cracks. J. Elasticity 4 (1974) 293-299.

32. S.W. Shaw, The dynamics of a harmonically excited system having rigid constraints. Parts I \& II, ASME J. Appl. Mech. 52 (1985) 453-464.

33. J. Aboudi, The dynamic indentation of an elastic half-space by a rigid punch. Int. J. Solids Structures 13 (1977) 995-1005. 